\title{
MULTICULTURALISMOS \& FRONTERA UNA CONSTRASTACIÓN ENTRE LAS POLÍTICAS Y LA REALIDAD DE LAS INSTITUCIONES EDUCATIVAS DE LA FRONTERA COLOMBO-VENEZOLANA
}

\section{MULTICULTURALISMS \& BORDER} A CONSTRASTATION BETWEEN POLICIES

\section{AND THE REALITY OF EDUCATIONAL INSTITUTIONS OF THE BORDER COLOMBO-VENEZOLANA}

\section{Torcoroma Velásquez Pérez ${ }^{1}$}

\section{Andrés Mauricio Puentes Velásquez ${ }^{2}$}

\section{Hugo Fernando Castro Silva ${ }^{3 s}$}

Universidad Pedagógica y Tecnológica de Colombia

Sogamoso, Boyacá, Colombia

$1 \quad$ tvelasquezp@ufpso.edu.co

Orcid: https://orcid.org/0000-0002-2968-2338 Universidad Francisco de Paula Santander seccional

Ocaña. Grupo de investigación GITYD Ocaña, Norte de Santander, Colombia

$2 \quad a m p u e n t e s v @ u f p s o . e d u . c o$

Orcid: https://orcid.org/ 0000-0003-2266-3131 Universidad Francisco de Paula Santander seccional

Ocaña. Grupo de investigación GITYD Ocaña, Norte de Santander, Colombia

3 s hugofernando.castro@uptc.edu.co Orcid: https://orcid.org/0000-0001-6020-402X

Universidad Pedagógica y Tecnológica de Colombia, Sogamoso, Boyacá, Colombia 


\section{RESUMEN}

La sociedad, en la actualidad enfrenta infinidad de cambios, transformaciones y obstáculos que han surgido del acontecer cotidiano, uno de ellos vinculado a la pandemia del COVID-19 (SARS-CoV-2); otros reflejado en el incremento del flujo migratorio que se vive en el país hermano Venezuela, donde las cifras no son nada alentadoras, y la crisis socio-económica cada vez se acentúa más; por tal razón, la presente investigación se plantea como objetivo: develar el impacto de los multiculturalismos y la constrastación de las políticas en las instituciones educativas de la frontera colombo - venezolana, eje de acción: Cúcuta, Villa del Rosario, La Parada (Colombia) y San Antonio de Táchira (Venezuela). Investigación realizada bajo los preceptos de la complementariedad metodológica (cuali-cuantitativos) apoyado en referentes ontológicos, epistemológicos y gnoseológicos basados en la interpretación, análisis y la reflexión (análisis hermenéutico), complementados con elementos de la estadística descriptiva (porcentajes), lo cual determina el nivel de impacto de los multiculturalismos en las realidades de la educación fronteriza y su relación las con políticas públicas educativas.

\section{PALABRAS CLAVE:}

multiculturalismos, frontera, políticas públicas educativas.

\section{ABSTRACT}

Society is currently facing countless changes, transformations and obstacles that have arisen from everyday life, one of them linked to the pandemic of COVID-19 (SARS-CoV-2); others reflected in the increase of the migratory flow that is experienced in the sister country Venezuela, where the figures are not at all encouraging, and the socio-economic crisis is becoming more and more accentuated; for this reason, the present research is proposed as an objective:
To reveal the impact of multiculturalism and the contrast of policies in the educational institutions of the Colombian-Venezuelan border, axis of action: Cúcuta, Villa del Rosario, La Parada (Colombia) and San Antonio de Táchira (Venezuela). Research carried out under the precepts of methodological complementarity (quali-quantitative) supported by ontological, epistemological and gnoseological referents based on interpretation, analysis and reflection (hermeneutic analysis), complemented with elements of descriptive statistics (percentages), which determines the level of impact of multiculturalisms in the realities of border education and its relation with educational public policies.

\section{KEY WORDS:}

multiculturalism, border, educational public policies.

\section{INTRODUCCIÓN.}

La sociedad se enfrenta a infinidad de cambios, transformaciones y avance a los cuales el entorno no es ajeno al dinamismo de la sociedad; razón que vale reflexionar en primer lugar a los efectos de la pandemia del COVID -19 (SARSCoV-2) y en el caso de la frontera a los aspectos que ha traído consigo el cierre de la frontera desde agosto del 2015; lo cual ha desatado aspectos inusuales como lo es el paso de un país a otro por las trochas (caminos verdes) de forma ilegal, lo cual ha arrastrado a su paso indicadores que no son lo más aceptables como por ejemplo: el narcotráfico, la trata de blancas, la prostitución, el secuestro entre otros. A ello se une el incremento del flujo migratorio entre Venezuela y Colombia, lo cual a su paso a traído efectos secundarios como lo es el incremento de la matrícula en las instituciones educativas que se encuentran en la frontera.

Aspectos que se encuentran respaldados en el informe técnico presentado por Fundaredes 
(2019) donde señala el: " $78 \%$ de los niños abandonaron la escuela en Venezuela y de los estudiantes que aún quedan matriculados, al menos $66 \%$ no asisten regularmente a las aulas, situación que sumada a $75 \%$ de abandono de los maestros a sus puestos de trabajo, evidencian un escenario histórico de crisis en el sistema educativo venezolano. A la fecha, más de 130 mil niños han salido de Venezuela, a estudiar en Colombia porque la escuela venezolana no ofrece las condiciones adecuadas. Así mismo, se puede asegurar que más de 9 mil niños cruzan la frontera a diario para estudiar en Colombia". (s/p). Sin duda alguna, es aquí donde se evidencia que la población migrante trae consigo infinidad de multiculturalismos con los cuales el docente debe tratar para mejorar la acción pedagógica en función de la formación de los niños y jóvenes migrantes.

Situación que se ha convertido en un nodo problemático; pues los docentes en ningún momento se han formado y capacitado para enfrentar mencionada situación; razón que requiere repensar el proceso de enseñanza y aprendizaje considerando develar el impacto de los multiculturalismos y la contrastación de las políticas en las instituciones educativas de la frontera colombo - venezolana, eje de acción: Cúcuta, Villa del Rosario, La Parada (Colombia) y San Antonio de Táchira (Venezuela). Pues es evidente que esos aspectos dejan ver como confluye la diversidad cultural en cada una de las instituciones educativas, de allí la insistencia por conocer en gran medida lo que son los multiculturalismos.

En la actualidad se observa con creciente preocupación que la migración ha generado una transculturización que en ocasiones se denomina transfronteriza; pues llegan de diversas latitudes geográficas y las instituciones educativas se convierten en centros donde confluyen y conviven; pues se observa como los dialectos de los niños demarcan las zonas de Venezuela (los zulianos son maracuchos, los del centro son los caraquistas y los andinos los llaman gochos). En efecto, esos aspectos conllevan a que los maestros se confundan cuando se enseña a niños de otras culturas, lo que permite un acercamiento al tema que se viene tratando y se muestra la introducción seguido del estado del arte, donde se presentan los antecedentes, bases teóricas - epistemológicas, seguido del marco contextual a ello se une los referentes metodológicos y se muestran los resultados, acompañado de la propuesta, seguido de la discusión y de las conclusiones, finalmente se muestra lo relacionado a las referencias bibliográficas.

\section{ESTADO DEL ARTE}

El estado del arte se encuentra definido por tres elementos base que son los antecedentes, las bases teóricas epistemológicas, luego se muestra el marco conceptual. De hecho, se muestra parte de los antecedentes, donde se presentan algunas experiencias y estudios investigativos vinculados con el tema, seguidamente se plantean las bases teóricas y epistemológicas, lo cual se caracteriza por mostrar parte de lo que es la parte conceptual seguida de los elementos gnoseológicos; ahora bien se muestra una descripción del marco contextual que encierra todo lo que es el eje fronterizo colombo - venezolano por la vertiente Cúcuta, Villa del Rosario, La Parada, (Colombia) y San Antonio del Táchira (Venezuela). Aspectos que confluyen en los siguientes párrafos:

\subsection{Antecedentes:}

En relación a los antecedentes es significativo mencionar que se realizó un acercamiento de los estudios más recientes existentes vinculados con lo que es los multiculturalismos y la frontera, razón por la cual se revisaron datas como Scopus, Web of Sciencie y Google Académico, donde se logró ver un grueso número de documentos online como tesis de pregrado, 
de postgrado (especialización, maestría y doctorado), artículos arbitrados, especializados y científicos; que sin duda alguna dejan ver una información muy valiosa representado en la siguiente figura:

Figura 1. Revisión de Data por concurrencia de palabras, autores y países.

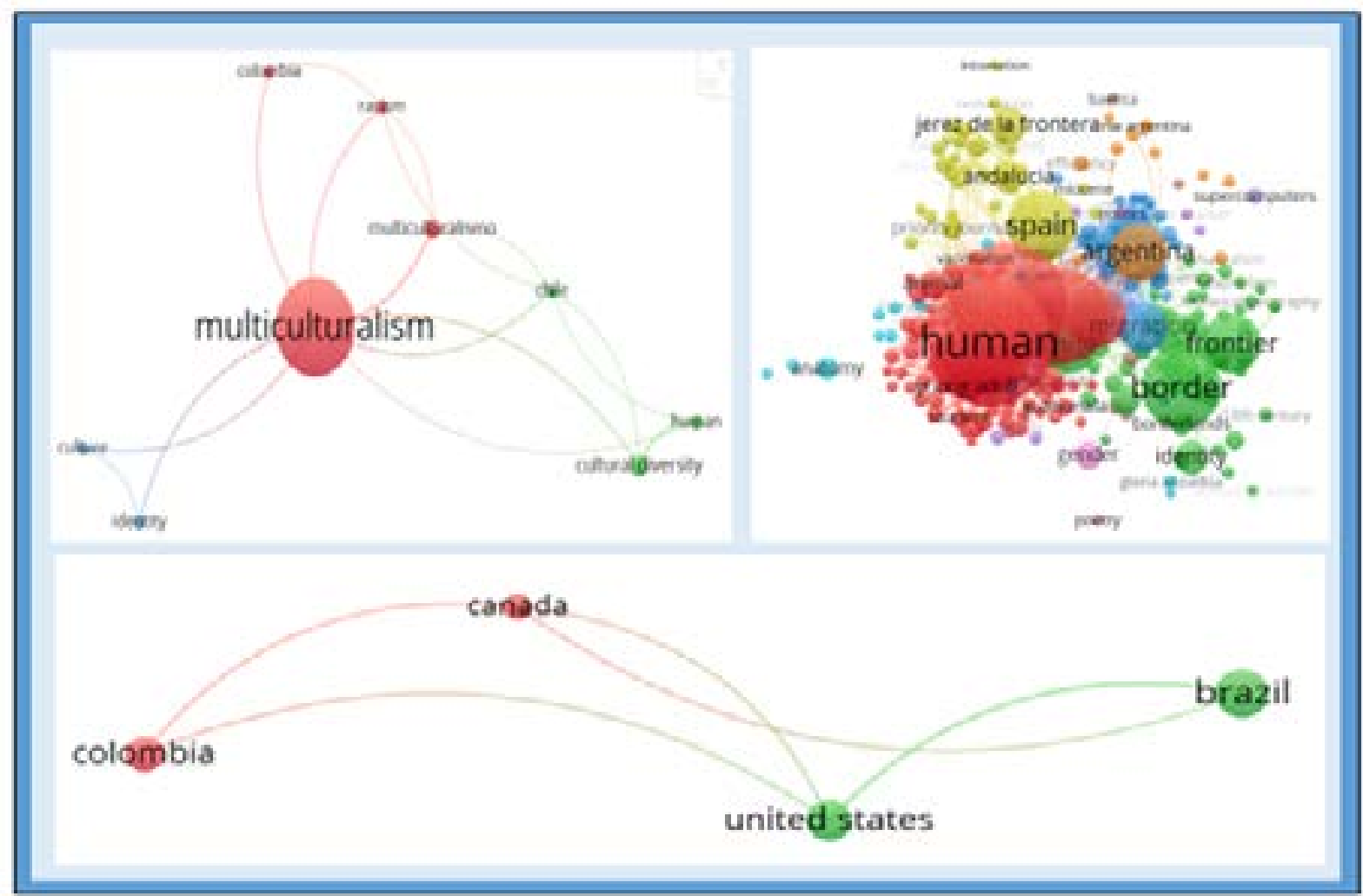

Fuente: Compilación y adaptación de Autores en Mapa de Datas - VOSviewer. 2021

Se logra observar en la figura 1. Unos mapas que muestran la concurrencia de palabras, autores, y así mismo se evidencia los países de mayor citación en los últimos cinco años, vale indicar que los países de mayor producción científica en relación a los multiculturalismos, y la frontera se encuentra Colombia, Canadá, Estados Unidos y Brasil. Es significativo mencionar que en esta ocasión mediante el apoyo del software Publish or Perish se logró determinar los siguientes trabajos, teniendo en cuenta los últimos años. De hecho, Barreto Anadon y Do Couto, (2020), realizaron un trabajo titulado: "Multiculturalismos E Formação Docente: Aproximações"; cuyo objetivo general se enmarco en; "Construir en el contexto de la formación del profesorado textos más democráticos, más inclusivos, menos etnocéntricos y menos prejuicios son solo algunos de los desafíos planteados por el multiculturalismo y que los planes de estudio que capacitan a los docentes tienen que lidiar permanentemente". (p.104).

Es un estudio realizado en Brasil y se deja ver en la Revista Didáctica Sistémica; donde se empleó una metodología cualitativa, interpretativa es importante señalar que se llegó a la conclusión según Barreto Anadon y Do Couto, (2020) que: "El multiculturalismo tal como lo entendemos es una propuesta de democratización que respeta e incluye los pluralismos, fundamentalmente éticos, ya que la 
cultura es variada y multiforme, que cuestiona los discursos de identidad esencialistas, que busca construir sociedades más justas e igualitarias". (p. 104). Concepción que se convierte en base de la presente investigación por la manera del cómo se refleja el multiculturalismo.

Ahora bien, ante dicha realidad es importante tener en cuenta el trabajo realizado por: Ledesma Correa, (2020) titulado: Multiculturalismos, procesos de comunicación intercultural y derechos humanos en los itinerarios de atención en salud del pueblo INY que vive en el municipio de Aruanã. Realizado en Brasil; cuyo objetivo se enmarco en que: "intenta aproximarse a una descripción sobre los diversos "multiculturalismos" y modalidades de comunicación intercultural que se presentan en los servicios de salud pertenecientes al Distrito de Saúde Araguaia". (p.1). (Ledesma Correa, 2020); los aportes encontrados en mencionado trabajo responden a una metodología cualitativa bajo los preceptos del método etnográfico.

En mencionado trabajo se llegó a concluir que: "Las políticas públicas en salud indígena promueven y aceptan la convivencia de diferentes culturas, como otras estrategias destinadas a gestionar el multiculturalismo que habita el Brasil, contienen preceptos éticos jurídicos universales, regionales y locales, para ofrecer un marco garantista de cumplimiento de lo reglamentado y a su vez fortalecer la cohesión social". (p. 11). Ledesma Correa, (2020). Sin duda, los multiculturalismos vistos desde ese posicionamiento teórico se pueden indicar que sirven de base para el trabajo que se viene planteando.

Ahora bien, se presenta la compilación de Albornoz Arias, N., Mazuera Arias, R., y Ramírez Martínez, C. (Ed.). (2018) quienes titulan su trabajo: Territorios: Frontera, migración y realidad social, en él se plantea: "aspectos particulares del fenómeno migratorio como perfil migrante, situaciones familiares, duelos, transformaciones familiares y sucesos negativos y positivos que le permiten entregar esta publicación que recoge una visión teórica y vivencial de que enriquece la intervención con población local y migrante a fin de promover elementos de resiliencia, emprenderismo y abordaje legal a un fenómeno de magnitudes globales". (p.7). Aspectos que permiten crear los fundamentos teóricos en relación a lo que se viene planteando.

Vale señalar que, en aporte teórico de Albornoz Arias, N., Mazuera Arias, R., y Ramírez Martínez, C. (Ed.). (2018) muestran aspectos de marcada relevancia, tal es el caso que se puede asegurar que: "la migración como eje nodal, no es posible abstraerse de la presencia de la violencia en el estudio de dicho fenómeno. A menudo forma parte de las complejas realidades que impulsan el deseo de cambiar de lugar de residencia, pero también, es una compañera de viaje indeseable durante ese proceso". (p. 7). En efecto mencionados elementos confluyen en un acercamiento de los diversos escenarios que se presentan en la frontera.

\subsection{Bases teóricas - epistemológicas:}

Con respecto al presente apartado es conveniente señalar que se manejan dos vertientes una encaminada en lo que es la parte conceptual teniendo presente que en este particular se manejan tres conceptos a saber: multiculturalismos, frontera y políticas públicas educativa; así mismo, se presenta los fundamentos gnoseológicos que responden a las teorías como lo es los modelos educativos, la teoría de la complejidad y por supuesto las teorías de la cultura. Vale señalar que son elementos muy explícitos; sin embargo, en este abordaje se trata de hacer una síntesis que permita la concreción para tener una visión sobre los elementos que se conjugan en relación al tema que se viene desarrollando. En tal sentido, se plantea los elementos conceptuales, tal como se muestran a continuación: 


\subsubsection{Multiculturalismos:}

Al referirse a los multiculturalismos, es necesario considerar que: "La diversidad cultural en un mundo cada vez más globalizado ha conducido a un gran uso (con el consiguiente desgaste) de términos como relativismo, pluralismo, multiculturalismo 0 interculturalidad. Sin embargo, a pesar de la cada vez más extensa literatura sobre el tema, pareciera que cada vez estamos más lejos de una noción más o menos compartida, ya que cada autor tiende a utilizar esos términos, $u$ otros afines, con un sentido propio". (p. 28). (Couder Cabral, 2009). Como se logra apreciar los multiculturalismos se convierten en según: Couder Cabral, (2009) en "un tipo particular de entramado político, no un ecosistema biótico-cultural. Si bien la metáfora es útil, conviene no olvidar que sólo es una metáfora. Abordar el multiculturalismo expresamente como una relación política nos permite comprender mejor las políticas públicas asociadas a él, en particular las educativas y lingüísticas". (p.31).

En efecto, el multiculturalismo se hace presente en la frontera; puesto que, confluye en un término que puede encerrar infinidad de elementos algunos positivos y otros mejorables que responden a las exigencias de la sociedad actual; pues quizá se convierte en aspectos donde se requiere la intervención social; puesto que, las modas, los dialectos, las formas de actuar se impregnan de todo lo que se va encontrando en el amigo y en ocasiones esos elementos se revierten en aspectos negativos para la sociedad y que a lo largo de frontera se logran develar cómo por ejemplo cómo el narcotráfico, la prostitución, la delincuencia organizada, entre otros han marcado el actual del hombre de la hombre y es allí donde aparece las instituciones educativas jugando un papel muy importante, tal es el que en estos recintos se observa con cierta preocupación el hacinamiento de estudiantes venezolanos en busca de mejoros condiciones de vida.

\subsubsection{Frontera:}

Se concibe como un espacio de intercambio de relaciones de poder, de elementos sociales, culturales, políticos y educativos que responde a un momento de la historia que se esté viviendo, el caso de la frontera colombo-venezolana ha dejado marcas históricas, pues en la actualidad se encuentra cerrada y aun así deja ver un dinamismo y movilidad de acciones que se pueden catalogar como actividades al margen de la ley, pues por los momentos no hay paso oficial. Por lo tanto, es preciso reflexionar sobre la concepción de la frontera que según: Brunet Jailly (2005) dice: "la noción de frontera ha experimentado cambios a lo largo de la historia, asimismo posee distintos significados y diversas acepciones por disciplina. Respecto al primer punto podemos afirmar que la historia de la idea de fronteras es amplia y retrotrae la antigüedad, pero claramente los contenidos han variado en el tiempo". (p.635).

Ahora bien, esta concepción permite plantear lo que Vera, Gonzales y Alejo (2011) afirman: "Los flujos migratorios cobran relevancia no solo porque modifican el número absoluto de personas que requieren el servicio educativo en cada lugar, sino también porque alteran la composición socio-demográfica de los lugares de origen y de destino. Por tanto, la migración condiciona la capacidad del sistema educativo para atender de manera eficiente y equitativa al conjunto de la población". (p.3). Realidad que conlleva a la reflexión de todo lo que se puede observar en las aulas de clase en los últimos tiempos, pues dentro de la frontera se observa infinidad de casos que pueden alterar la tranquilidad a raíz que se empiezan a construir casas temporales en las zonas vulnerables, debajo de los puentes, canchas, entre otras y sin duda alguna eso conlleva a que se altere la convivencia pacífica eso conlleva a que dentro 
de la población de migrantes se estimule el pensamiento de supervivencia y ante un estímulo surge entonces una reacción, lo cual conlleva a las conductas se alteren y en ocasiones surgen acciones fuera de lo común que conlleva a la alteración del entorno. Por lo tanto, pareciera que en gran medida los diversos efectos se adhieren a la parte de la cultura de los migrantes y es allí donde surge la intervención del docente para mediar y orientar a los niños y jóvenes que asisten a las instituciones educativas de la frontera para que la convivencia sea mejor.

\subsubsection{Políticas públicas educativas:}

En relación a las políticas públicas educativas es pertinente señalar que las mismas se encuentran definidas en lo que establece la Constitución Política Colombia de 1991 en sus artículos 67 y 68; y sus características son recogidas en los planteamientos de: Robledo Castro, C., Amador Pineda, L. H., \& Ñáñez Rodríguez, J. J. (2019): "La emergencia de políticas encaminadas a mejorar la calidad de la educación se repite en una serie de retos para la formación de maestros, en especial del educador infantil. Este último es un agente crucial para la consecución de las metas trazadas en las políticas públicas y educativas de primera infancia [...], lo que le demanda la necesidad de desarrollar nuevos conocimientos disciplinares que involucren el análisis y la comprensión de la realidad de la escuela [...] Sin embargo, a pesar de que su formación se considera una apuesta clave para el desarrollo integral de la primera infancia [...], esto no ha redundado en un mayor reconocimiento social de la labor del educador infantil ni en mejores oportunidades para su desenvolvimiento profesional". (p. 172).

Razón que conlleva a reflexionar en torno a buscar alguna política educativa que permita la atención de los inmigrantes en la frontera; sin duda alguna eso conlleva a que el docente puede armarse de estrategias, y herramientas para que puedan generar modos de enseñanza entendiendo y comprendiendo los multiculturalismos, eso permite que la UNESCO citado en Robledo Castro, C., Amador Pineda, L. H., \& Ñáñez Rodríguez, J. J. (2019) quienes señalan: "La idea es apostar por fortalecer el campo profesional de la educación para la primera infancia con criterios que impulsen una agenda y políticas fundamentadas en un crecimiento con calidad, garantizar condiciones materiales y simbólicas específicas, y así lograr el posicionamiento a partir de la fuerte convicción de que el empoderamiento y la autonomía de un campo profesional del educador infantil se sustentan en una base de conocimiento común, muy especializado, construido y legitimado participativamente por sus miembros". (p. 173).

\subsection{Fundamentos gnoseológicos de los multiculturalismos en la frontera:}

En relación a los fundamentos gnoseológicos de los multiculturalismos en la frontera se debe indicar que se hace énfasis en los modelos pedagógicos de enseñanza que se emplean en las instituciones escolares y se logró indagar que los docentes se valen de un modelo de enseñanza holístico que incluye lo mejor de lo que el aprendizaje significativo (Ausubel), donde los estudiantes de acuerdo a sus intereses se vinculan con los temas que más les guste. Adicional a ello es importante tener presente que también se considera lo mejor del aprendizaje por descubrimiento (Jerome Brunner) con la intención que los estudiantes puedan adquirir conocimientos, así mismo, se presenta el modelo constructivista (Vygotsky); modelo que implica ir construyendo los aprendizajes para alcanzar un buen conocimiento.

Bajo la misma mirada es conveniente señalar que dentro de lo que es las teorías que respaldan la cultura se encuentra la teoría de la complejidad (Edgar Morín) que se encarga de sobre manera entender y comprender lo que rodea a los estudiantes inmigrantes que por sus características definen lo que es lo dinámico y 
complejo de la cultura, teniendo en cuenta que a ello se une lo relacionado a la axiología y a la diversidad cultural; pues es importante indicar que la cultura es la que demarca el actuar y desenvolvimiento de los inmigrantes; puesto que, las estadísticas superan las expectativas y por tal razón es conveniente tener un respaldo ontológico que permita entre la relación del ser con la sociedad.

\subsection{Marco Contextual:}

En ese contexto, colombo - venezolano el espacio fronterizo se calcula en $2219 \mathrm{Km}$. de longitud, aproximadamente (Tovar. 1991); Allí convergen una serie de aspectos socioeconómicos, políticos, jurídicos y culturales que lo diferencian del resto del país, pues se desenvuelve una situación que tiene sus propias normas y leyes. En un estudio de las fronteras venezolanas (Tovar 1991) organiza la frontera colombo-venezolana en dos sectores; en uno de ellos se ubican por el lado venezolano los Estados Apure y Amazonas y colombiano la región Arauca-Vichada-Vaupués; en el otro se localizan los Estados Táchira y Zulia y los departamentos de la Guajira, Magdalena y Norte de Santander. Estos últimos de Colombia; es importante señalar que en este caso se plantea el trabajo investigativo en el eje fronterizo Cúcuta, Villa del Rosario, La Parada (Colombia) y San Antonio del Táchira (Venezuela); que sin duda alguna es la parte de la frontera que diariamente recibe a miles y miles de venezolanos, quienes escapan de una crisis socio-económica que nadie explica, pero de lo que si se está seguro es que la frontera aparece cerrada desde el 2015 y cada día son más los caminos verdes que surgen se dice que aproximadamente existen unas 200 trochas para pasar el Río Táchira, quien es el que divide a ambas naciones.

\section{REFERENTES METODOLÓGICOS.}

La presente investigación responde a lo que en la actualidad se denomina complementariedad metodológica (cuali-cuantitativos) pues según Martínez (2005), citado por Blanco, Neligia, \& Pirela, Johann (2016) quienes señalan que: "la complementariedad sirve para explicar la incapacidad humana de agotar la realidad con una sola perspectiva, punto de vista, óptica o abordaje y por ende, el uso de diversos enfoques produce una significativa riqueza al conocimiento obtenido, ya que logra integrar en un todo coherente, los aportes de diferentes personas, filosofías, métodos y disciplinas". (p.99). En este caso enmarcado en los enfoques de investigación cualitativa y cuantitativa, basado en los referentes ontológicos, epistemológicos y gnoseológicos apoyados en el paradigma interpretativo, bajo los preceptos teóricos del análisis hermenéutico (aplicación del circulo hermenéutico) y dicha información se respalda con algunos elementos de la estadística descriptiva (porcentajes) que dejan manejar informaciones avaladas por cantidades numéricas.

De hecho, Blanco, Neligia, \& Pirela, Johann (2016) al citar a Bericat (1998) define: la complementariedad como una estrategia de integración para producir conocimiento a través una práctica múltiple para aproximarse a los datos con el propósito de superar la posición unidimensional y divisoria de los enfoques, evaluando los aportes de cada uno en el proceso de investigación para la comprensión y/o explicación del objeto de estudio". (p. 100). Aspectos que se logran concretar en la presente investigación, pues se combinan técnicas, instrumentos y uno que otro procedimiento con la intención de tener un acercamiento al fenómeno de investigación que se viene planteando.

\section{RESULTADOS.}

Enfunción de los resultados es importante señalar que existen dos vertientes uno encaminado a la parte cualitativa donde fue necesario valerse de la observación y de la aplicación de estudios hermenéuticos a documentos y hechos que se 
presentan en la línea fronteriza; vale señalar que dicho procedimiento se realizó a través de la aplicación del software ATLAS ti lo cual genera la redes semánticas de las categorías trabajadas que son los multiculturalismos, la frontera y las políticas públicas educativa. En tal sentido, la segunda vertiente se encamina en elementos cuantitativos apoyados en el procesamiento estadístico de elementos descriptivos representados en porcentajes.

Por lo tanto; a continuación, se muestra la contrastación teórica reflejada en las tres categorías con sus respectivas definiciones, con la intención de canalizar acciones para develar los rasgos culturales de todas aquellas personas que a diario llegan a la frontera en busca de mejores condiciones de vida; es importante señalar que se platea una matriz con la finalidad de mostrar ordenadamente lo concerniente a lo que va a originar las redes semánticas y por supuesto los argumentos que respaldan el conocer el impacto de los multiculturalismos en lo que es la implementación de las políticas públicas educativas en la frontera.

Tabla 1. Contrastación teórica de las categorías previas y reportes de categorías emergentes.

\begin{tabular}{|l|l|l|}
\hline Categorías previas & \multicolumn{1}{|c|}{ Definición } & Categorías emergentes \\
\hline Multiculturalismos & $\begin{array}{l}\text { Se definen como los rasgos que } \\
\text { caracterizan a las personas en } \\
\text { relación a los elementos culturales, } \\
\text { es decir su dialecto, forma de } \\
\text { desenvolverse y desempeñarse } \\
\text { en el entorno. }\end{array}$ & - Multiculturalismo factual \\
- Multiculturalismo normativo \\
\end{tabular}




\begin{tabular}{|c|c|c|}
\hline Frontera & $\begin{array}{l}\text { Es la línea imaginaria que delimita } \\
\text { los linderos de dos países, donde } \\
\text { se muestra el paso de un país a } \\
\text { otro y en el caso de la frontera que } \\
\text { se refiere al estudio se encuentra } \\
\text { cerrada por uno de los países y } \\
\text { eso conlleva a escenarios inéditos. }\end{array}$ & $\begin{array}{l}\text { Efectos Positivos: } \\
\text { - Busca de mejores condiciones } \\
\text { de vida } \\
\text { - Bienestar social } \\
\text { - Acceso a la educación } \\
\text { - Acceso a programas sociales } \\
\text { - Acceso a ayuda humanitaria } \\
\text { Efectos negativos: } \\
\text { - Narcotráfico } \\
\text { - Prostitución } \\
\text { - Trata de blancas } \\
\text { - Delincuencia organizada } \\
\text { - Alteración del orden público } \\
\text { - Secuestro } \\
\text { - Cobro de vacuna }\end{array}$ \\
\hline Políticas Públicas Educativas & $\begin{array}{l}\text { Se definen desde lo que establece } \\
\text { la Constitución Política de } 1991, \\
\text { seguido de la Ley } 115 \text { de } 1994, \\
\text { donde se establecen las leyes } \\
\text { y normas para que se logre } \\
\text { consolidar una educación de } \\
\text { calidad }\end{array}$ & $\begin{array}{l}\text { - Aceptación de estudiantes } \\
\text { extranjeros } \\
\text { - Beneficios de becas } \\
\text { - Beneficios de programas } \\
\text { sociales } \\
\text { - Acceso a programas de salud }\end{array}$ \\
\hline
\end{tabular}

Fuente: Autores, 2021

La tabla 1, indica cuales son las categorías emergentes que vienen a definir lo que son los códigos previos o en su defecto in vivo que permiten un acercamiento a los elementos que se viven cotidianamente en la sociedad; partiendo de allí, lo que son las redes semánticas y luego se corrobora con elementos cuantitativos reflejado en lo que es datos estadísticos que corroboran los argumentos ya descritos. De hecho, se logra observar como: "moradores de la zona de la parada abordan a quienes van a cruzar la frontera por los caminos verde con fajos de dinero (venezolano) ofreciendo comprar pesos y quienes e hacen llamar los maneros" (Obs.01, 2021). Así mismo, es pertinente mostrar otra escena en el mismo lugar "personas con acento caraqueño abordan a las mujeres que tienen el pelo largo y les ofrecen una cantidad de pesos por su cabello" (Obs.02, 2021) y no se puede dejar a un lado: "quienes ofrecen comprar celulares, computadores canaimas, laptops, entre otros" (Obs.03, 2021). Como se 
puede apreciar de las observaciones se logra obtener información valiosa para interpretar lo que se presenta en la línea fronteriza, es bueno hacer notar que no solamente allí se encuentran venezolanos, sino que también hacen vida otras nacionalidades. Sin duda alguna, todos esos elementos confluyen en la siguiente figura número 2:

Figura 2. Red Semántica de las Categorías Tratadas

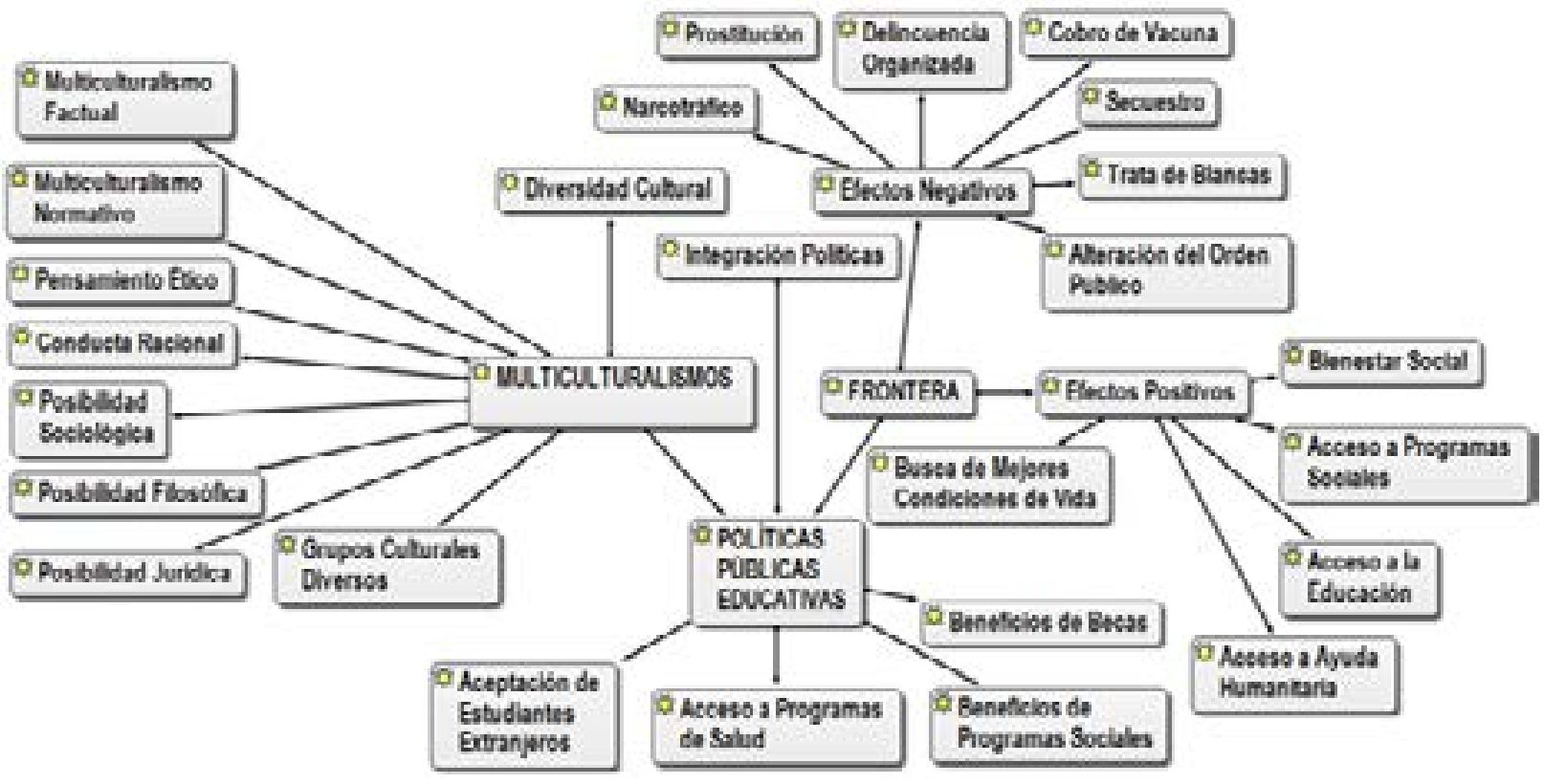

Fuente: Material compilado, sobre las categorías base - Autores, 2021

Se logra evidenciar en la red semántica como se relacionan las categorías multiculturalismos, frontera y políticas públicas educativas, lo cual converge en mostrar parte de lo que se vive en las zonas de frontera, donde los inmigrantes se encuentran con elementos susceptibles de estudio donde se logra contrastar la información encontrada y desde ese posicionamiento teórico surgen nuevos elementos que se deben tener muy en cuenta para poder ofrecer algunos aspectos que permiten abrir los caminos para generar propuestas académicas que conlleven a mejorar la situación que se logra develar en la frontera que en ocasiones es dejada a un lado sin interesar que es una situación muy importante que puede mejorar el hacinamiento social en las instituciones educativas.

Aunado a ello vale indicar que, según Angarita de la Secretaría De Educación, (información Estoy en la Frontera, 2021) manifestó que: "La matrícula de estudiantes provenientes de Venezuela en Norte de Santander en los últimos tres años ha ido crecimiento vertiginosamente, especialmente en Cúcuta. Hoy son 20.106 alumnos venezolanos que están estudiando en los 40 municipios del departamento. Cúcuta y Villa del Rosario tienen la mayor cantidad de niños en aula. Son 12.946 alumnos, de los cuales la capital Norte santandereana alberga 
9.317 y Villa 3.629". (s/p). Como se puede apreciar en estos datos se logra develar que los datos estadísticos reflejan un comportamiento de migrantes sin precedentes los cuales han conducido a que la matrícula en las instituciones de la frontera haya aumentado y eso conlleva a que el maestro debe tratar con una diversidad cultural enorme para la cual en ocasiones el docente no se encuentra preparado.

\section{PROPUESTA.}

En función a la propuesta es conveniente tener presente que después de analizados los diferentes aspectos que se enmarcan en el impacto de los multiculturalismos en la frontera, es pertinente plantear algunas acciones que conlleven a que se logren plantear algunas acciones pedagógicas para que se puedan empezar a concientizar desde las instituciones educativas, teniendo presente que con esas acciones se busca la armonía en las instituciones y en el entorno donde viven los niños y jóvenes inmigrantes, en tal sentido, se plantea la siguiente tabla:

Tabla 2. Acciones para fortalecer los multiculturalismos en la frontera desde la perspectiva pedagógica.

\begin{tabular}{|c|l|}
\hline Acción pedagógica & \multicolumn{1}{c|}{ Descripción } \\
\hline $\begin{array}{c}\text { Campañas de Promoción } \\
\text { y Difusión }\end{array}$ & $\begin{array}{l}\text { Las campañas se realizan mediante las redes virtuales y por supuesto } \\
\text { en físico; pues dentro de ello se emplea la infografía virtual, los posters } \\
\text { pedagógicos, los brouchure pedagógicos, los banners entre otros. Adicional a } \\
\text { ello se crean documentos que se facilitan a los interesados como las normas } \\
\text { de convivencia para que sean conocimiento de quienes forman parte de la } \\
\text { población que se encuentra en la situación de inmigrantes. }\end{array}$ \\
\hline \multirow{5}{*}{$\begin{array}{c}\text { Talleres virtuales y } \\
\text { presenciales }\end{array}$} & $\begin{array}{l}\text { Desde las instituciones educativas se emplean los talleres virtuales y } \\
\text { presenciales sobre lo que es las normas de convivencia, la forma de cómo } \\
\text { el docente debe tratar a los estudiantes que son de otra nacionalidad, así } \\
\text { como también estos talleres deben ir dirigidos a los padres, representantes } \\
\text { y acudientes para que ellos entiendan y comprendan cómo debe ser el trato } \\
\text { entre semejantes a pesar de existir las diferencias culturales. }\end{array}$ \\
\hline Webinar sobre los & $\begin{array}{l}\text { Se debe implementar la técnica del webinar para que se logre concretar } \\
\text { grupos de WhatsApp y telegram para estar en constante formación con } \\
\text { respecto a lo que son los multiculturalismo y la forma y manera como se } \\
\text { deben tratar a quienes forman parte de inmigrantes, igualmente es importante } \\
\text { tener en cuenta que se logre una constante asesoría sobre los derechos } \\
\text { humanos, entre otros. }\end{array}$ \\
\hline Tertulias virtuales & $\begin{array}{l}\text { Las tertulias son espacio que se crean a través de las redes para que } \\
\text { se realicen contacto online con quienes tengan dudas, opiniones y por } \\
\text { supuestos sugerencias para que cada día exista un tema a desarrollar y se } \\
\text { logre dar apoyo desde las instituciones educativas. }\end{array}$ \\
\hline Eanal de yuotube & $\begin{array}{l}\text { El canal de youtube se debe crear con la finalidad de mostrar algunos } \\
\text { posicionamientos teóricos existentes con respecto a lo que son los } \\
\text { multiculturalismos y por supuesto eso conlleva a que se esté en constante } \\
\text { difusión de temas importantes relacionados con los multiculturalismo, y su } \\
\text { impacto entre los habitantes de la frontera. }\end{array}$ \\
\hline
\end{tabular}

Fuente: compilación y adaptación, autores, 2021 


\section{DISCUSIÓN.}

En cuanto a este apartado es significativo mencionar que la información encontrada, según la observación y los datos manejados de estadísticas e informes especializados; en tal sentido, se logró ver que la frontera es visitada por diversas culturas, algunas personas usan la línea fronteriza para ir en busca de otros destinos que mejore las condiciones socio-económicas de vida, (tal como lo ven en la actualidad los venezolanos); sin embargo, otros solo llegan a la frontera y allí se quedan pues lo presupuestado no les alcanzo para seguir avanzando; por lo tanto, es oportuno tener en cuenta que todos esos aspectos se adhieren a la personalidad de los habitantes de la frontera y es allí donde aparecen las salidas fáciles y muchos jóvenes y personas adultas son reclutados por las fuerzas de poder y los conviertes en su trabajadores en actividades de tipo ilegal y a pagados a muy bajo precio (Obs.03, 2021).

De hecho, Tapia, M. (2017), al citar a Benedetti, (2013). Señala que la frontera es la "Implantación más bien puntual o real, es construida con los dispositivos políticos, culturales, económicos y materiales, generalmente puestos sobre el límite, allí en el frente, para efectivizar el control ante situaciones actuales o potenciales de egreso/ingreso al territorio: es un filtro para lo que se mueve a través del límite". (p. 66-67). Vale indicar que la dinámica en la frontera es muy compleja pareciera que cada vez se van adjuntando más y más aspectos y en su mayoría se observa con insistencia cómo elementos negativos; razón por la cual se debe considerar que hacer desde las instituciones educativas para que los maestros puedan enfrentarse a esa complejidad de culturas que se deben tratar con mucho cuidado y tolerancia para mantener un clima de armonía en las instituciones que se encuentran en el eje fronterizo.

Aunado a ello, es pertinente señalar que Heyman, (2012) citado en Tapia, M. (2017); pues se plantea: "Las fronteras son escenarios de una serie de movimientos expresados en cruces diarios, semanales o por días que no tienen por objetivo el establecimiento en el "otro lado". Por lo tanto, la noción de movilidad es más apropiada para comprender los movimientos en torno o través de las fronteras porque es más amplia y comprende más aspectos que la migración". (p.73). Partiendo de dicha realidad, es importante señalar que Arriaga Rodríguez, (2012) señala: "la frontera como espacio socialmente construido es de aparición relativamente reciente, pues es producto del giro teórico y metodológico ocurrido en la geografía [...] En términos generales, los autores de estos movimientos conciben a la frontera como un espacio cambiante, de manera que la definición del concepto es construida por las prácticas sociales y no por la categoría geográfica con la cual se la vincula". (p.73).

\section{CONCLUSIONES.}

Al cumplir con cada una de las fases de investigación se logra determinar que el objetivo fue cumplido; pues este caso se enmarca en develar el impacto de los multiculturalismos y la contrastación de las políticas en las instituciones educativas de la frontera Colombo - Venezolana, eje de acción: Cúcuta, Villa del Rosario, La Parada (Colombia) y San Antonio de Táchira (Venezuela). Es importante indicar que los multiculturalismos dejar ver aspectos positivos y otros mejorables; tal es el caso que los aspectos mejorables pues superan los positivos; puesto que, se logra ver narcotráfico, secuestro, prostitución, trata de blancas, delincuencia organizada, entre otros.

Así mismo, se observa con creciente preocupación que el número de matrícula ha aumentado en las instituciones educativas de la frontera, pues muchos niños los escriben en las instituciones educativas por un plato de comida o en su defecto por disfrutar de algunos beneficios que no los logran obtener si los 
niños no están inscritos formalmente. Adicional a ello es conveniente señalar que el docente debe actualizarse y prepararse para poder enseñar a este tipo de alumnos (migrantes), pues es oportuno tener en cuenta que a los multiculturalismos se unen los elementos antes señalados (narcotráfico, secuestro, prostitución, trata de blancas, delincuencia organizada, entre otros), puesto que, en ocasiones el sistema no sabe cómo responder cuando observa que algunos venezolanos reaccionan a full carrera (a pie) para alcanzar el transporte que se dirige al paso fronterizo con la finalidad de ofrecer a sus semejantes a pasar sus cosas por las trochas y a cambio esperan alguna recompensa pedagógica.

En ocasiones se observa como otras personas pagan alguna recompensa económica para que los pasen por encima del río alzados, sin duda alguna, es una situación muy difícil y atípica; de hecho, eso viene cargado de incertidumbre, miles de personas transitan por los caminos verdes (200 trochas) en busca de sustento, mejores condiciones de vida y en la mayoría de los casos esos caminos son utilizados para escapar de una crisis que les ha marcado y dejan atrás familia, sueños y amigos que quizá por cuestiones del destino les deben abandonar para buscar un futuro mejor y garantizarle a quienes se quedan una mejor forma de vida, pues eso viene impregnado de culturas y de elementos visionarios que tal vez les pueda ayudar a cambiar las condiciones socioeconómicas de vida.

\section{REFERENCIAS BIBLIOGRÁFICAS}

Albornoz Arias, N., Mazuera Arias, R., y Ramírez Martínez, C. (2018). Territorios: Frontera, migración y realidad social. Colombia: Ediciones Universidad Simón Bolívar. https://bonga.unisimon.edu.co/bitstream/ handle/20.500.12442/3281/Territorios_ PDF.pdf?sequence $=1$ \&isAllowed $=y$
Estoy en la Frontera, (2021). ¿Cuántos niños venezolanos estudian en Norte de Santander?. Colombia. https:// estoyenlafrontera.com/educacion/cuantosninos-venezolanos-estudian-en-norte-desantander

Arriaga Rodríguez, (2012). El concepto de frontera en la geografía humana. Perspectiva Geográfica 17, 71-96.

Dialnet-EIConceptoFronteraEnLaGeografi aHumana-5626943.pdf

Barreto Anadon y Do Couto, (2020). Multiculturalismos E Formação Docente: Aproximações. Revista Didáctica Sistémica. 22(2), 103-116. https://www. seer.furg.br/redsis/article/view/11691/8804

Blanco, Neligia, \& Pirela, Johann, (2016). La complementariedad metodológica: Estrategia de integraciónde enfoques en la investigación social. Espacios Públicos, 19(45), 97-111. https://www.redalyc.org/ articulo.oa?id=67646966005

Brunet-Jailly, E. (2005). Theorizing Borders: An Interdisciplinary Perspective. Geopolitics, 10(4), 633-649.

Couder Cabral, (2009) Multiculturalismo y educación. Revista Cultura y representaciones sociales. http://www. scielo.org.mx/pdf/crs/v4n7/v4n7a2.pdf

Fundaredes (2019). Violación Al Derecho A La Educación Por Colapso Del Sistema Educativo Venezolano Obliga A Los Niños A Estudiar En Colombia, Resultando Su Desplazamiento Hacia Escenarios Hostiles. (Prensa Fundaredes). https://www. fundaredes.org/2019/09/09/fundaredes78-de-los-ninos-abandonaron-la-escuelaen-venezuela/ 
Ledesma Correa, (2020). Multiculturalismos, procesos de comunicación intercultural y derechos humanos en los itinerarios de atención en salud del pueblo INY que vive en el municipio de Aruanã. https://www. revistas.ufg.br/racs/article/view/65684/356

Robledo Castro, C., Amador Pineda, L. H., \& Ñáñez Rodríguez, J. J. (2019). Políticas públicas y políticas educativas para la primera infancia: desafíos de la formación del educador infantil. Revista Latinoamericana de Ciencias Sociales, Niñez y Juventud, 17(1), 169-191. https:// dx.doi.org/10.11600/1692715x.17110

Tapia, M. (2017). Las fronteras, la movilidad y lo transfronterizo: Reflexiones para un debate [Borders, mobility and the transborder space: reflections for a discussion] Estudios Fronterizos, 18(37), 61-80, doi:10.21670/ref.2017.37.a04

Tovar L. R. (1991). "La Nueva Ciencia". Revista del Centro de Investigaciones Geodidácticas, (2)3, enero-junio, 87-118.

Vera, Gonzales y Alejo (2011). Migración y educación Causas, efectos y propuestas de cambio para la situación actual de migración escolar. Programa de Investigación Estratégica en Bolivia. La Paz. Bolivia. 